\title{
HABILIDADES SOCIOEMOCIONALES DE LOS DOCENTES Y EL APRENDIZAJE COOPERATIVO DE LOS ESTUDIANTES DEL NIVEL AVANZADO UN CENTRO DE EDUCACIÓN BÁSICA ALTERNATIVA PARA PRESIDIARIOS.
}

\section{SOCIOEMOTIONAL SKILLS OF TEACHERS AND COOPERATIVE LEARNING OF ADVANCED LEVEL STUDENTS A CENTER FOR ALTERNATIVE BASIC EDUCATION FOR PRESIDIARIES.}

\author{
Juana Rosa Santana Pilco ${ }^{1}$ \\ Julio Carrasco Rosado²
}

Aceptado: $17 / 09 / 2019$

Publicado online:15/01/2020

\begin{abstract}
RESUMEN
El propósito de este estudio fue determinar la relación de las habilidades socioemocionales de los docentes y el nivel de aprendizaje cooperativo, caso de los estudiantes del CEBA Noé Moisés Dávalos Ybañez - Tacna, año 2015. En la metodología se aplicó un tipo de investigación observacional, prospectivo de cohorte transversal. La muestra fue conformada por 19 docentes y 67 estudiantes del CEBA Noé Moisés Dávalos Ybañez - Tacna. El instrumento, para los docentes se consideró el test de Bar-On, que evalúa las habilidades socioemocionales. Se usó un cuestionario dirigido a los estudiantes, para evaluar el aprendizaje cooperativo. En lo referente al alcance de la investigación, ha sido enfocado a evaluar la inteligencia emocional y el aprendizaje cooperativo, para proporcionar sugerencias que las instituciones educativas lo puedan desarrollar; evidenciando la relevancia la formación integral, a través de la dimensión afectiva. Se ha encontrado que el desarrollo de las habilidades socioemocionales se encontró en un nivel promedio en el $68.4 \%$ de los docentes, el nivel de aprendizaje cooperativo de los estudiantes es promedio con un $40,3 \%$, y que existe diferencia significativa de las habilidades intrapersonales, interpersonales, de adaptabilidad, del manejo del estrés y del estado de ánimo general de los docentes con los niveles de aprendizaje cooperativo de los estudiantes del nivel avanzado del
\end{abstract}

\footnotetext{
${ }^{1}$ Magíster en Docencia Universitaria Y Gestión Educativa, Docente en Educación, Tacna, Perú

${ }^{2}$ Magíster en Educación con mención en Administración Educacional, Docente de la Facultad de Educación y Ciencias de la Comunicación y Humanidades de la Universidad Privada de Tacna, Tacna, Perú
} 
CEBA. Se concluye que existe relación significativa de las habilidades socioemocionales de los docentes con el nivel de aprendizaje cooperativo de los alumnos del nivel secundario del CEBA Noé Moisés Dávalos Ybañez Tacna.

Palabras claves: Aprendizaje cooperativo, Habilidades socioemocionales.

\begin{abstract}
The purpose of this study was to determine the relationship between the socioemotional skills of teachers and the level of cooperative learning of the students of the secondary level of CEBA Noé Moisés Dávalos Ybañez - Tacna, 2015. In the methodology, a type of observational, prospective research was applied. transverse cohort. The sample was made up of 19 teachers and 67 students. The instrument, for teachers, was considered the Bar-On test, which assesses social-emotional skills. A questionnaire aimed at students, to evaluate cooperative learning. With regard to the scope of research, research has focused on evaluating emotional intelligence and cooperative learning, to provide suggestions that educational institutions can develop; evidencing the relevance of the integral formation, through the affective dimension. It has been found that the development of socio-emotional skills is at a promedio or average level in $68.4 \%$ of teachers, the level of cooperative learning of students is regular with $40.3 \%$, and that there is a significant difference in the intrapersonal, interpersonal skills, adaptability, stress management and general mood of teachers with cooperative learning levels of students at the advanced level of the CEBA. And it is concluded that there is a significant relationship between the socioemotional skills of the teachers and the level of cooperative learning of the students of the secondary level of CEBA Noé Moisés Dávalos Ybañez - Tacna.
\end{abstract}

Keywords: Social-emotional skills, cooperative learning.

\title{
INTRODUCCIÓN
}

Se ha considerado habilidades socioemocionales de los docentes, y de acuerdo a lo planteado por Bar-On (1997), fundamenta la inteligencia emocional como la agrupación de habilidades interpersonales, personales y emocionales, las que inciden por la vida diaria de las personas, donde pueda hacerle frente a presiones del contexto social. El ser inteligente es fundamental, para alcanzar la superación en la vida. En tal sentido, el modelo de Bar-On (1988) trabajó con cinco componentes fundamentales (interpersonal, manejo del estrés, intrapersonal, adaptabilidad y ánimo general) por lo que las habilidades socioemocionales de los docentes han sido relacionadas con el aprendizaje cooperativo, que fueron estudiados para el análisis en los estudiantes, acerca de su inteligencia emocional. En el campo de la educación a nivel internacional, la Organización de las Naciones Unidas para la Educación, la Ciencia y la Cultura (Organización de las Naciones Unidas para la Educación, la Ciencia y la Cultura, 1998), en su informe Delors (1997), le da gran relevancia a lo que significa las emociones y resalta la necesidad de educar el componente emocional de la persona junto a su 
dimensión cognitiva, que permita el fortalecimiento las habilidades socioemocionales. La gran dificultad que se percibe para el aprendizaje de adultos, es que las características de la población estudiada no se presentan con las mismas condiciones, ya que el estudiante adulto al cual este estudio se enfoca se encuentra en calidad de presidiario, de alguna manera, por diversas situaciones se encuentran recluidos en una cárcel, algunos pagando una condena perpetua, otros con algunos años de reclusión. Dentro de los factores causales de esta problemática, está en las habilidades socioemocionales. Muchos profesores lamentablemente no poseen habilidades interpersonales, es decir, que no se relacionan fácilmente con sus colegas ni con sus estudiantes; provocando lastimosamente poca comunicación, en su entorno laboral. Vivas, Chacón y Chacón, (2010), concluye que los docentes deben practicar el saber ser y el saber convivir, para ello resulta indispensable desarrollar su inteligencia emocional. Aguayo-Cotallat y Nuñez-Espinoza (2012) refiere que el aprendizaje cooperativo contribuye al éxito académico. Burguillos-Peña (2015) concluye en la urgencia de que los docentes desarrollen una adecuada inteligencia emocional, que implique la mejora de sus habilidades socioemocionales. Menacho-López (2010) afirma que en el uso del método de aprendizaje cooperativo, logró mejorar el rendimiento académico, en comparación al método de enseñanza tradicional. Asimismo, Guevara (2014), concluye que las estrategias de aprendizaje cooperativo ha confirmado la mejora comprensión de textos. Limaymanta (2014) opina que los docentes muchas veces no poseen las suficientes habilidades socioemocionales, como interpersonales, la empatía, relaciones interpersonales y la responsabilidad social; así como la destreza de adaptabilidad, en cuanto a la terminación de problemas, estudio de la realidad y la variabilidad, precisamente como el dominio del estrés, como la consideración al estrés y el dominio de los impulsos. Mamani (2015) concluyó en que la eficacia de la experiencia cooperativa contribuye a alcanzar el logro de competencias de los estudiantes. Herrera (2016) concluye finalmente que las competencias socioemocionales de los docentes son insuficientes, porque el promedio de 25 indicadores fue 57,37. Y el aprendizaje cooperativo de los alumnos es falto de eficacia, dado que el promedio resultó de 20,50. Goleman (1996) considera que los principales componentes de la inteligencia emocional son Autoconocimiento emocional, Autocontrol emocional (o autorregulación), Automotivación y Reconocimiento de emociones ajenas (o empatía). La Teoría de Mayer Caruso y Salovey (2000), establece "que la inteligencia emocional es la capacidad de ver y expresar sentimientos con precisión, de hacer sentimientos que permitan el pensamiento; y para comprender y supervisar los sentimientos, que promueve la mejora emocional y académica. Además, es significativo demostrar que los modelos e instrumentos que dependen de la aptitud dependen de la primera conceptualización de la inteligencia emocional (Salovey \& Mayer, 1990), (Mayer, Dipaolo, \& Salovey, 1990). La inteligencia emocional es un conjunto de habilidades inherentes a las personas y que les permite actuar en su vida diaria con éxito, ya que se basa en que son perceptivos, expresivos, capaz de asimilar situaciones complejas (Mayer, Caruso, \& Salovey, 2000). Modelos basados en características o modelos combinados (Roberts, Zeidner, \& Matthews, 2001), sostienen que la construcción de la inteligencia emocional es más extensa y considera las aptitudes identificadas con la preparación y utilización de sentimientos y capacidades individuales. El Modelo de Vallés y Vallés (1999) describe 
una variedad de habilidades, que componen la inteligencia emocional, enumerándolas éstas en diferentes aspectos: Tolerar la autofrustación, identificar los sucesos que provocan emociones positivas y negativas, conocerse a sí mismo, motivarse uno mismo, llegar a acuerdos razonables con compañeros y compañeras, conocer identificar lo que resulta fundamental en cada condición, de reforzarse uno mismo, contener la ira en situaciones de provocación, mostrarse optimista, controlar los pensamientos, y hablarse uno mismo para orientar la conducta, rechazar peticiones poco razonables, serán más dialogantes, valorarán opiniones de los demás, se divertirán, y harán tareas más agradables, sonreirán, tendrán confianza en sí mismo, mostrar ser dinámico y actividad, comprender los sentimientos de los demás. (Vallés \& Vallés, 1999). El aprendizaje cooperativo es una preparación instructiva en la que existe tal organización para darse cuenta, que los grupos heterogéneas de estudios pueden cooperar para lograr un objetivo que corresponda en un proceso de aprendizaje similar. Las virtualidades potenciales exhibidas por los sistemas de aprendizaje agradables se refieren solo a la mejora que parecen ofrecer en el esfuerzo por aprender entre los individuos de la clase. (Santos-Rego, Lorenzo-Moledo, \& Priegue-Caam, 2009). Es importante lo que el instructor logra para persuadir a los estudiantes. (Casillas, 2001). El uso de técnicas que hacen mayor hincapié en aspectos positivos, brindará a esos procedimientos una mayor aceptación en todo tipo de intervenciones (Davidson., 1995). La dimensión de desarrollo de habilidades afectivas es comprenderse a sí mismos, comprender y tolerar a otros. (García, 2001). El aprendizaje cooperativo es "un mecanismo colaborador que desea desarrollar hábitos de trabajo en equipo, la solidaridad entre compañeros, y que los alumnos intervengan autónomamente en su proceso de aprendizaje" (Liberman, 2000).

\section{OBJETIVOS}

a) Identificar el nivel de desarrollo de las habilidades socioemocionales, aprendizaje cooperativo y medir la relación entre las habilidades intrapersonales de los docentes y el nivel de aprendizaje cooperativo de los estudiantes del nivel avanzado del CEBA Noé Moisés Dávalos Ybañez, Tacna 2015.

b) Medir la relación entre las habilidades interpersonales de los docentes, habilidades de adaptabilidad, habilidades del manejo del estrés y estado de ánimo general según el nivel de aprendizaje cooperativo de los estudiantes del nivel avanzado del CEBA Noé Moisés Dávalos Ybañez, Tacna 2015.

\section{METODOLOGÍA}

Investigación Básica, observacional de corte transversal retrospectivo. El ámbito fue en la CEBA Noé Moisés Dávalos Ybáñez, Tacna, año 2015. Las unidades de estudio son 19 docentes y 67 estudiantes del nivel secundario del CEBA Noé Moisés Dávalos Ybáñez de Tacna, año 2015. La recolección de la información se hizo en forma personal y de primera fuente. Se aplicó el cuestionario Test de inteligencia emocional de ICE:NA de Bar-On de Ugarriza \& Pajares (2004) para docentes de - Tacna, año 2015 y el cuestionario denominado Aprendizaje cooperativo de los estudiantes del nivel avanzado elaborado por la investigadora. 


\section{RESULTADOS}

El 68.4\% de los docentes muestran un nivel de habilidades socioemocionales promedio, y el $15.8 \%$ muestra niveles bajos y en la misma proporción evidencian un nivel de habilidades alta. Aclaramos que no hubo ningún docente con muy bajo nivel ni con muy alta nivel de habilidades socioemocionales.

Tabla 1

Niveles de habilidades socioemocional total de los docentes del CEBA Noé Moisés Dávalos Ybáñez

\begin{tabular}{clcc}
\hline & & $\mathrm{n}$ & $\%$ \\
\hline & Muy baja & 0 & $0.0 \%$ \\
& Baja & 3 & $15.8 \%$ \\
Habilidades & Promedio & 13 & $68.4 \%$ \\
Socioemocionales Total & Alta & 3 & $15.8 \%$ \\
& Muy alta & 0 & $0.0 \%$ \\
& Total & 19 & $100.0 \%$ \\
\hline
\end{tabular}

La habilidad socioemocional mejor manejada por los docentes está en el componente de manejo del estrés, en tanto que los demás componentes se encuentran por debajo del promedio. Se observar también que el componente peor percibido por los docentes es el de adaptabilidad, seguido del componente estado de ánimo general. Sin embargo, como se aprecia en la figura 1 no existe diferencia significativa entre los niveles de desarrollo entre los cinco componentes de las habilidades socioemocionales a excepcion del componente adaptabilidad y manejo del estrés.

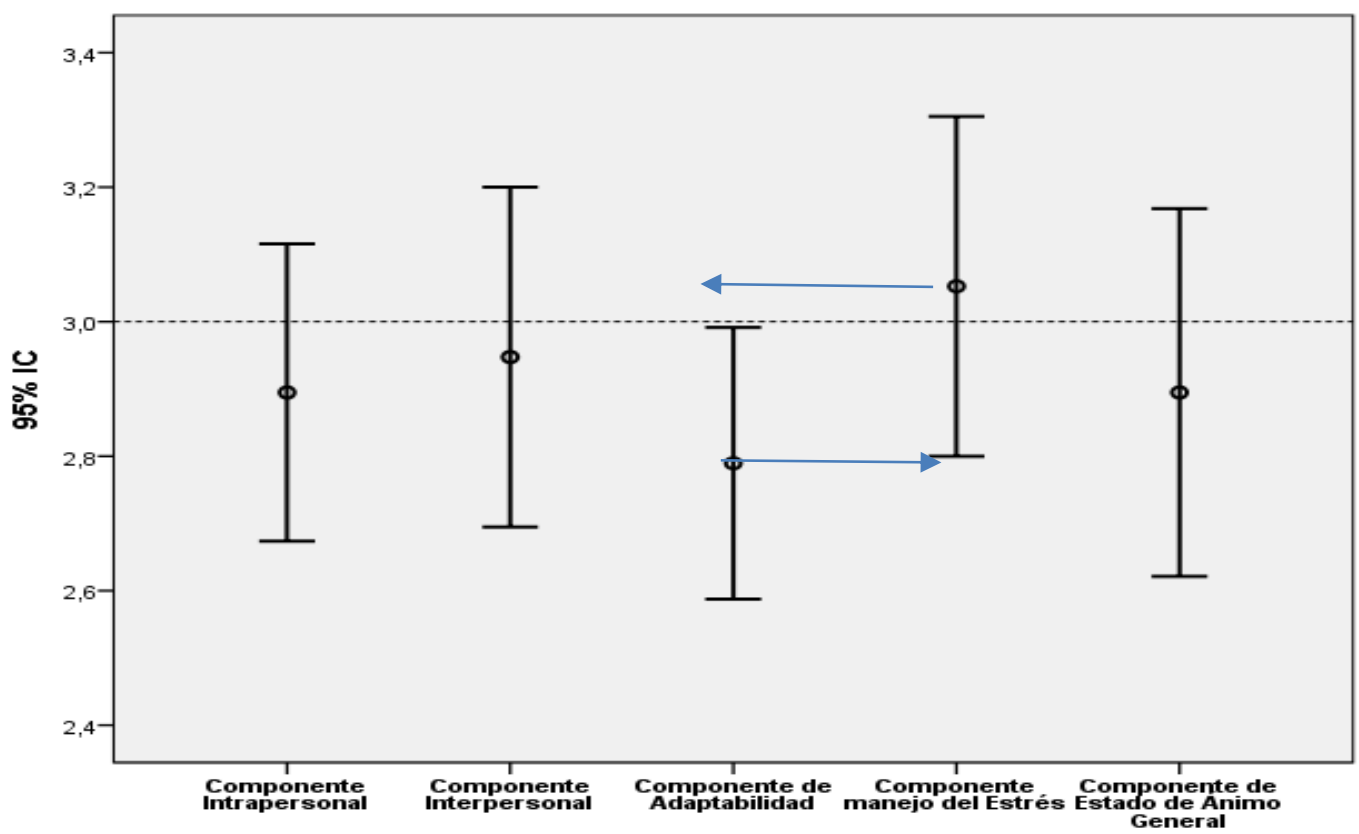

Figura 1

Desarrollo de habilidades socioemocionales por componentes. 


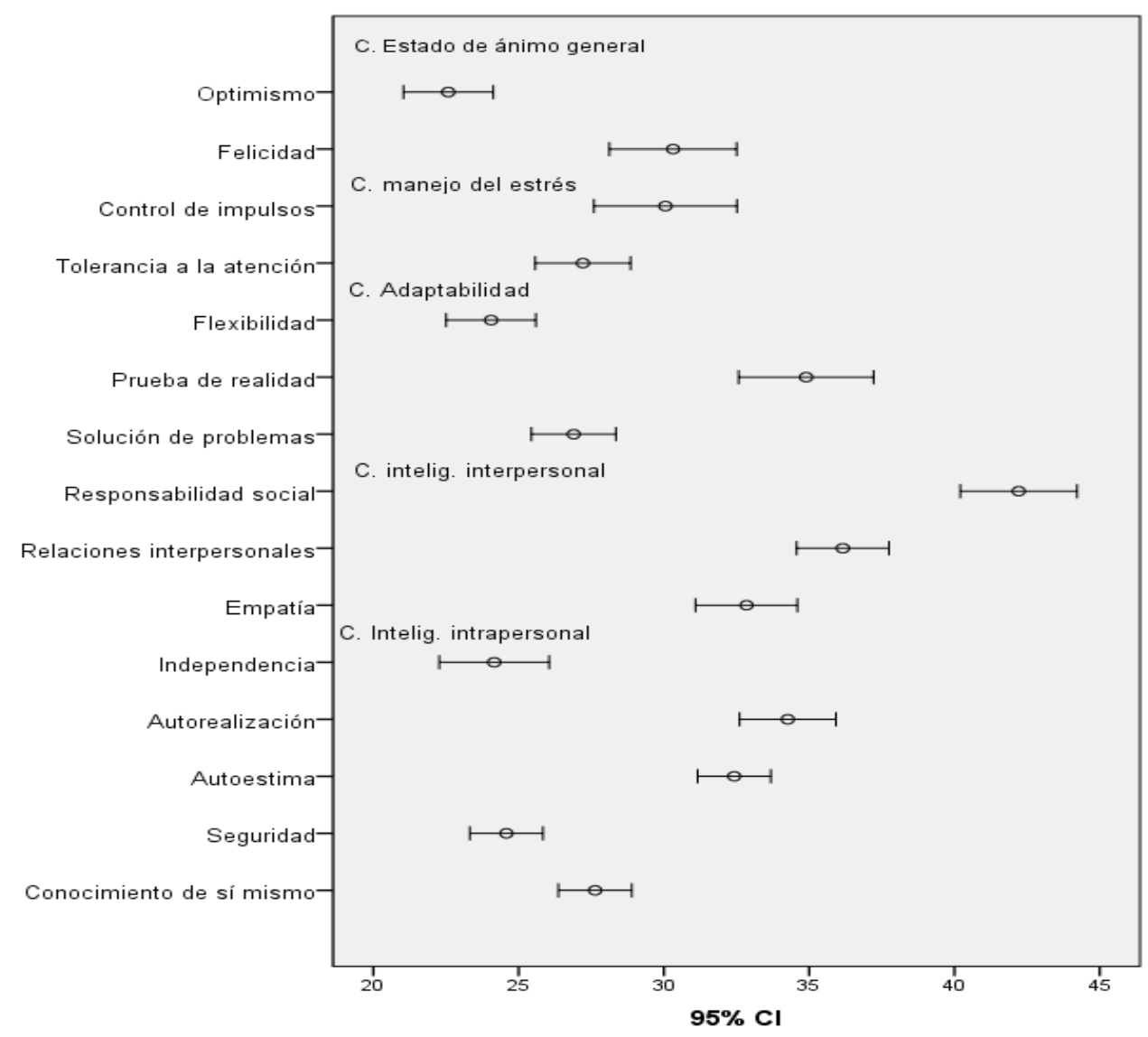

\section{Figura 2}

Indicadores por dimensiones de las habilidades socioemocionales de los docentes.

En la figura 2 se muestra que en la inteligencia intrapersonal lo peor percibido es la independencia y seguridad de sí mismo, en la inteligencia interpersonal lo peor prcibido es la empatía, en cuanntoa adaptabilidad lo peor percibido es la flexibilidad, en el manejo del estrés lo mejor percibido es el control de los impulsos y en el estado deánimo general se observa falta de optimismo.

La tabla 2 muestra los niveles de aprendizaje cooperativo que fueron evaluados en un total de 67 estudiantes en condición de carcelería, en Tacna. Donde se observa que para el $40.3 \%$ el nivel de aprendizaje cooperativo fue regular, seguido del $37.3 \%$ percibieron un nivel inadecuado de aprendizaje cooperativo y solo para el $22.4 \%$ el nivel de aprendizaje cooperativo.

\section{Tabla 2}

Distribución porcentual según nivel de aprendizaje cooperativo percibido por los estudiantes del CEBA.

\begin{tabular}{cccc}
\hline & & $\mathrm{n}$ & $\%$ \\
\hline \multirow{3}{*}{ Nivel de Aprendizaje } & Nivel inadecuado & 25 & $37.3 \%$ \\
Cooperativo & Nivel regular & 27 & $40.3 \%$ \\
& Nivel adecuado & 15 & $22.4 \%$ \\
& Total & 67 & $100.0 \%$ \\
\hline
\end{tabular}


En la figura 3 se presenta los niveles de aprendizaje cooperativo percibido desde las habilidades socioemocionales de los docentes, de acuerdo a las tres dimensiones evaluadas. Así se observa que para el $35.8 \%$ de los estudiantes el aprendizaje cooperativo por interdependencia positiva fue inadecuado, pero para el $31.3 \%$ fue regular y un $32.8 \%$ sí fue adecuado. En cuanto a la dimensión responsabilidad individual tuvo un nivel inadecuado en el $56.7 \%$ de los estudiantes seguido de un nivel adecuado para el $22.4 \%$ y regular en el $20.9 \%$. Con respecto a la dimensión interacción potenciadora para el $52.2 \%$ el nivel fue inadecuado, seguido del $29.9 \%$ que opina que sí fue adecuado y para el $17.9 \%$ el nivel fue regular.

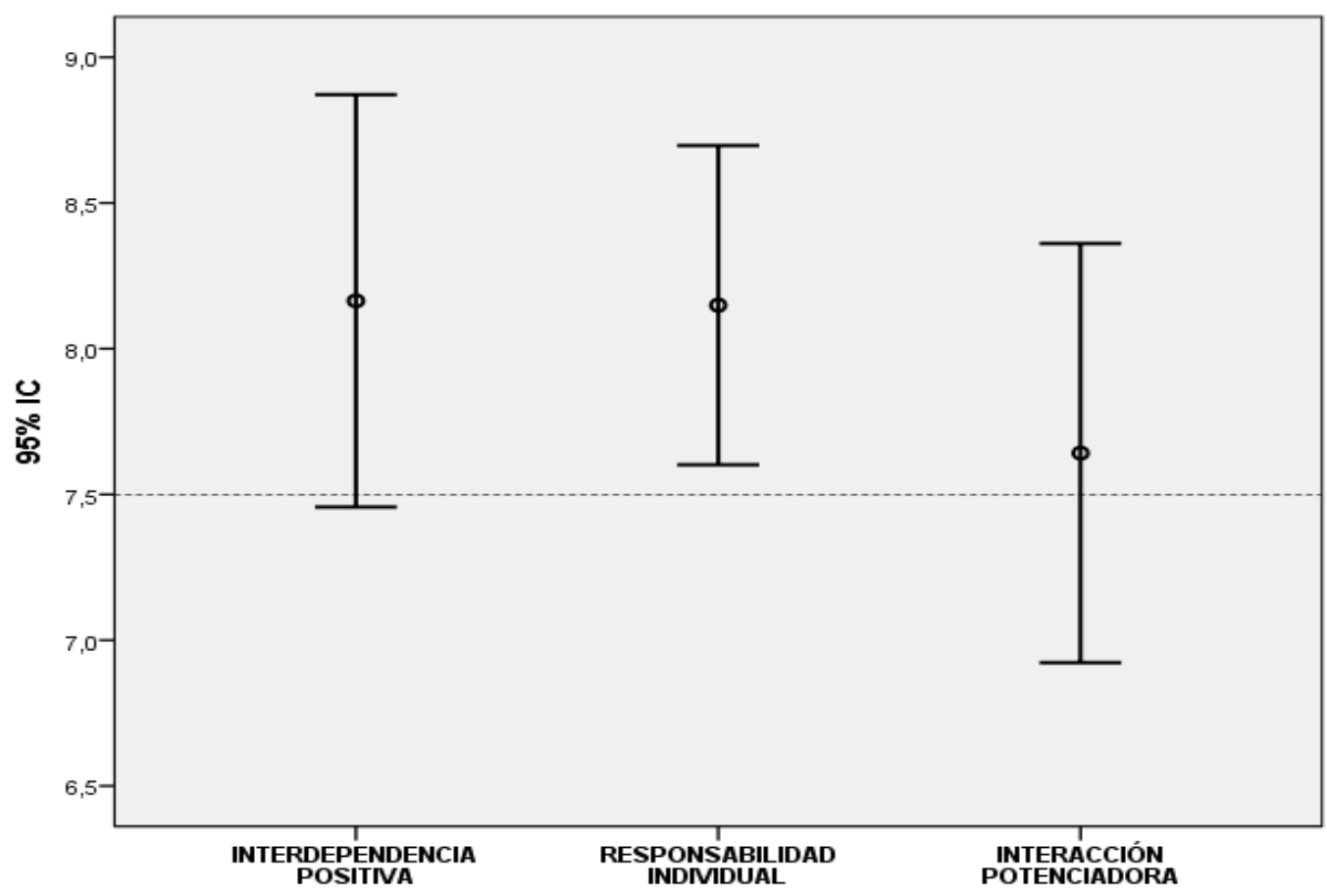

Figura 3

Identificación de las dimensiones del aprendizaje cooperativo según percepción de los estudiantes del CEBA

En la figura 4 se observa el comportamiento de las dimensiones aprendizaje cooperativo. Lo mejor desarrollado por los estudiantes fue la responsabilidad individual, seguido de la independencia positiva, en tanto que lo peor valorado fue la interacción potenciadora. Si se tuviera que tomar decisiones sería en cuanto a la interacción potenciadora primordialmente. 


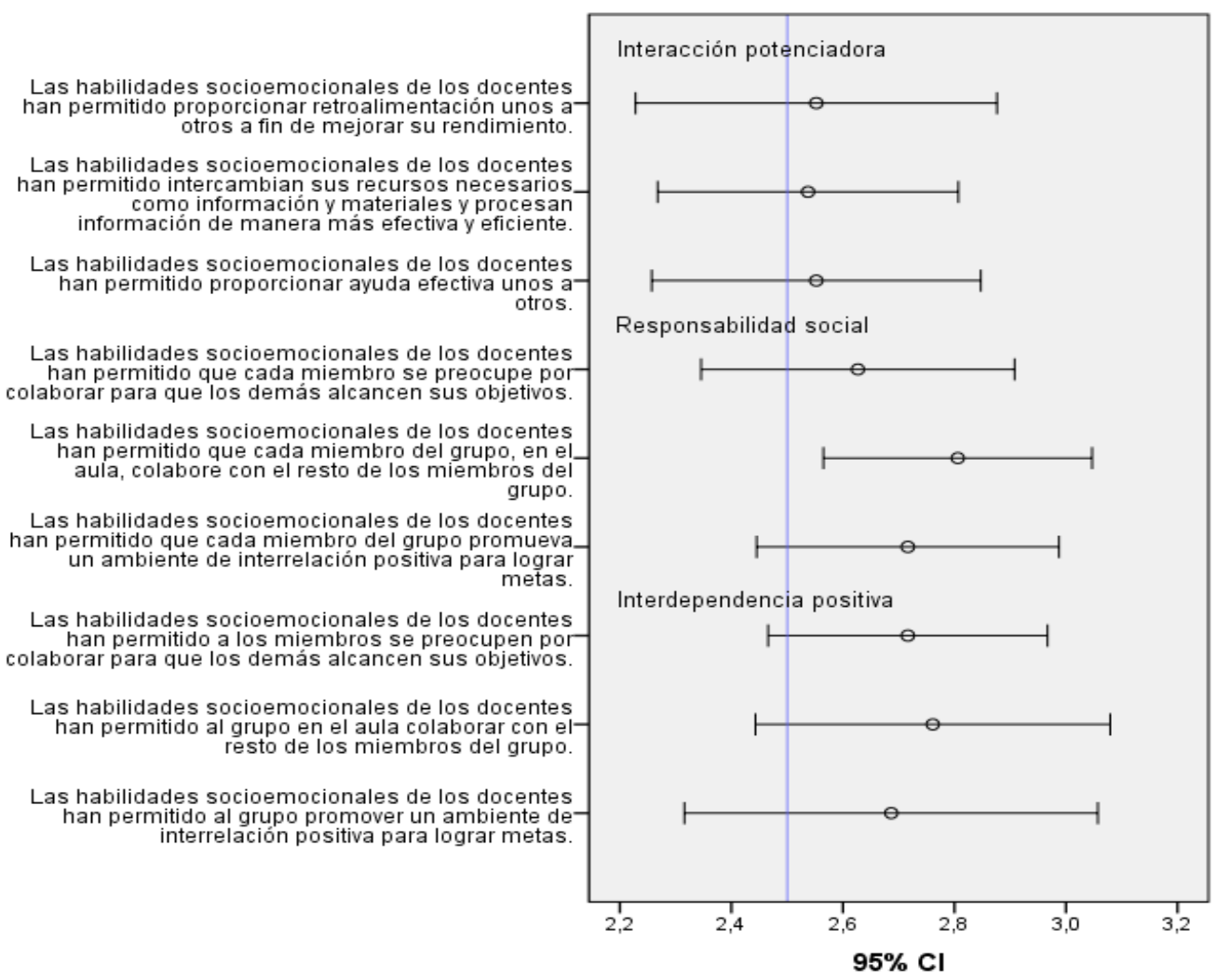

Figura 4

Indicadores por dimensiones del aprendizaje cooperativo de los estudiantes.

Existen diferencias significativas entre las habilidades socioemocionales de los docentes y el aprendizaje cooperativo de los estudiantes (Tabla 3 )

\section{Tabla 3}

Relación significativa entre las habilidades socioemocionales de los docentes y el nivel de aprendizaje cooperativo de los estudiantes

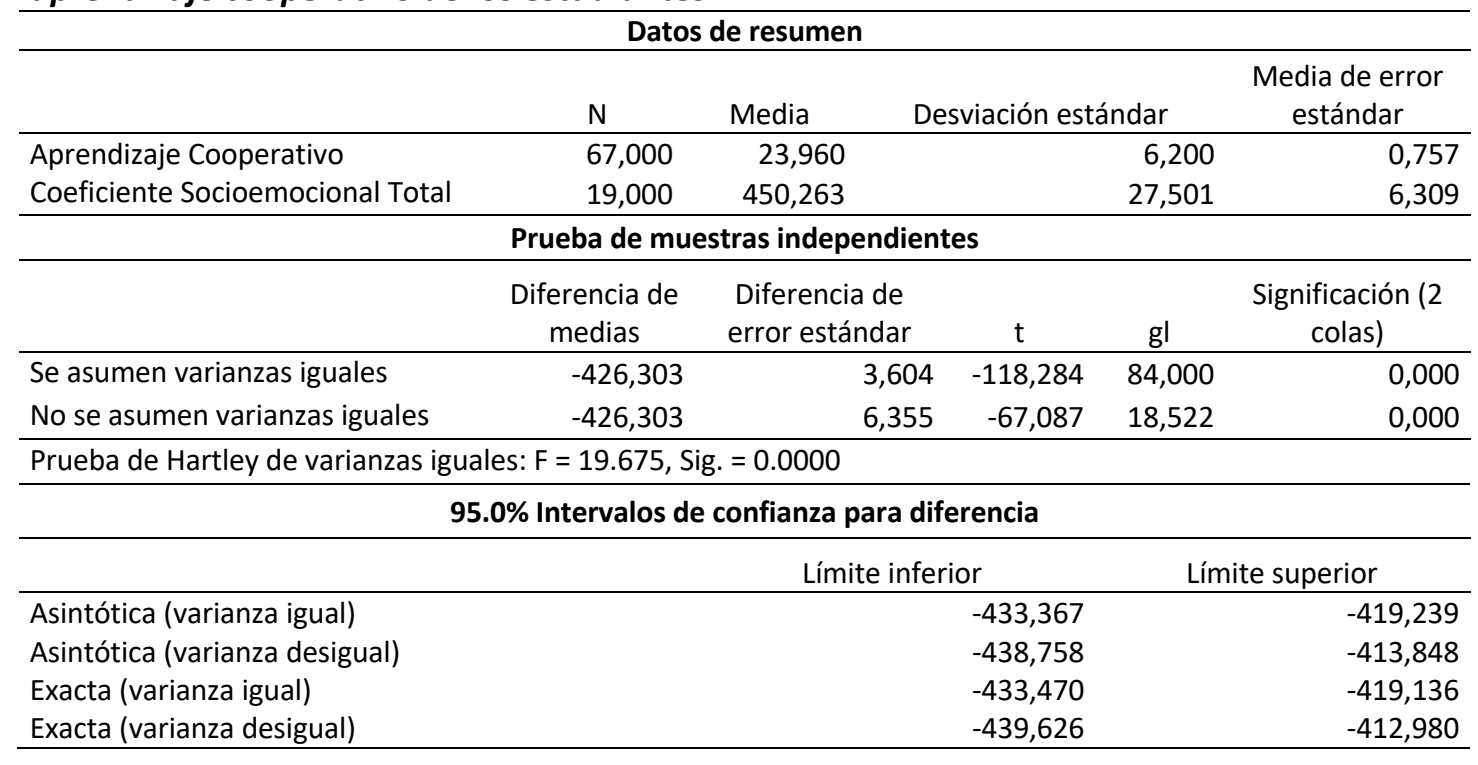




\section{DISCUSIÓN}

El desarrollo de las habilidades socioemocionales se encuentra en un nivel promedio en el $68.4 \%$ de los docentes que laboraron en el Centro de Educación Básica Alternativa Noé Moisés Dávalos Ybañez, donde los estudiantes están en condición de carcelería. El nivel de aprendizaje cooperativo de los estudiantes es regular con un 40,3\%, para el $37.3 \%$ fue inadecuado y para el $22.4 \%$ fue adecuado. Se ha establecido la relación entre las habilidades intrapersonales de los docentes y el nivel de aprendizaje cooperativo de los estudiantes ( $p: 0,000$ ). Las habilidades de adaptabilidad de los docentes ( $p: 0,000)$, las habilidades del manejo del estrés ( $p: 0,000)$ y ánimo general de los docentes ( $p$ : 0,000 ) están asociadas al nivel de aprendizaje cooperativo de los estudiantes. Se recomienda a la Dirección disponga la implementación de un Programa de intervención psicopedagógica de fortalecimiento de las habilidades socioemocionales y fortalezcan las prácticas pedagógicas en los docentes.

\section{REFERENCIAS BIBLIOGRÁFICAS}

Aguayo-Cotallat, J. E., \& Nuñez_Espinoza, M. (2012). Aprendizaje cooperativo y su incidencia en el rendimiento académico de los estudiantes de segundo año de Bachillerato especialidad Ciencias del Instituto Tecnológico Pelileo. Tesis de maestría, Universidad Técnica de Ambato, Ecuador. Obtenido de

Arce, M. (2016). Aprendizaje cooperativo y los hábitos de estudios en los estudiantes del Instituto de Educación tecnológico Aeronáutico- 2016. Tesis de maestría, Universidad César Vallejo.

Ato, M., López, J. J., \& Benavente, A. (2013). Un sistema de clasificación de los diseños de investigación en psicología. . Anales de Psicología, 29(3), 1038-1059.

Avila, L. (2018). Formulación De La Hipótesis. Recuperado el 2 de febrero de 2019, de

Bar-On, R. (2000). Emotional and Social Intelligence:Insights from the Emotional Quotient Inventory. San Francisco: Jossey -Bass.

Burguillos-Peña, A. I. (2015). La inteligencia emocional y el sentido de coherencia en la percepción de la salud de los docentes universitarios. Tesis de doctorado, Universidad de Huelva, España.

Carrasco, S. (2009). Metodología de la Investigación científica. Lima: Editorial San Marcos. 1ra Reimpresión.

Casillas. (2001). Aprendizaje Cooperativo para Modelado Lingüístico Flexible Basado en Reglas Difusas: Interpretabilidad y Precisión. Universidad de Granada. España: Tesis doctorales en red.

Davidson., N. (1995). International perspectives on cooperative and collaborative learning. International Journal of Educational Research, 23, 197-200.

Delors, J. (1997). La Educación es un Tesoro Escondido. Distrito Federal, México: Ediciones UNESCO. Obtenido de https://www.rau.edu.uy/docs/delors_s.pdf

Dewey. (1967). Experiencia y Educación. Buenos Aires: Losada.

Díaz-Aguado, M. J. (2003). Educación interculural y aprendizaje cooperativo. Madrid: Pirámide. 
Estrada, M., Monferrer, D., \& Moliner, M. (2016). El Aprendizaje Cooperativo y las Habilidades Socio-Emocionales: Una Experiencia Docente en la Asignatura Técnicas de Ventas. Formación universitaria, 9(6), 43-62.

Fernández - Berrocal, P., \& Ruiz, D. (2008). La Inteligencia emocional en la Educación. . Revista Electrónica de Investigación Psicoeducativa ISBN 1696-2095, 6(2), 421436.

Ferreiro, R., \& Calderón, M. (2007). El ABC del aprendizaje cooperativo. México: Trillas.

Fragoso Luzuriaga, R. (2015). Inteligencia emocional y competencias emocionales en educación superior, ¿ un mismo concepto?. Revista Iberoamericana de Educación Superior, 6(16),

Fundación Santillana para Iberoamérica. (2014). ¿Educamos para la vida? Inteligencia emocional. Una mirada práctica. (X. P. 2013, Ed.) Colombia: Editorial Santillana S.A.

García, M. (2001). Entrenamiento en competencias. Encuentros en Psicología Social, 1 (3), 27-32.

Goleman, D. (1996). Inteligencia Emocional. Barcelona- España: Kairós.

Guevara, M. F. (2014). Estrategias de Aprendizaje Cooperativo y Comprensión Lectora con textos filosóficos en Estudiantes de Filosofía de la Facultad de Educación de la Universidad Nacional Amazónica de Madre de Dios, año 2012. Tesis de maestría, Universidad Mayor de San Marcos.,

Herrera, H. (2016). Competencias socioemocionales de los docentes y el aprendizaje cooperativo de los estudiantes de la Facultad de Ciencias Jurídicas y Empresariales de la Universidad Nacional Jorge Basadre Grohmann - Tacna, año 2014.

Johnson, D., \& Johnson, R. (2014). Cooperative Learning in 21st Century. . Anales de Psicología, 30(3), 841-851.

Johnson, D., Johnson, R., \& Holubec, E. (1999). El aprendizaje cooperativo en el aula. Barcelona: Paidós.

Kagan, S. (1994). Cooperative Learning. . San Juan Capistrano, California: Kagan Cooperative Learning.

Lara. (2005). El aprendizaje cooperativo: un modelo de intervención para los programas de tutoría escolar en el nivel superior. Revista de la Educación Superior, vol. XXXIV (1), núm. 133, enero-marzo, 2005, pp. 87-104. Obtenido de

Liberman, A. (2000). Schools as Collaborative Cultures: creating the Future Now. Pensylvania: The Falmer Press, U.S. .

Limaymanta, K. E. (2014). Resiliencia e inteligencia emocional en estudiantes de secundaria de Instituciones Educativas del Distrito de Huancayo. Tesis de maestría, Universidad Nacional del Centro del Perú., Unidad de Posgrado de la Facultad de Educación, Huancayo.

Lobato, C. (1997). Hacia una comprensión del aprendizaje cooperativo. Revista de Psicodidáctica, núm. 4, 1997, pp. 59-76.

Mamani, M. A. (2015). Aprendizaje cooperativo y logro de competencias en las estudiantes del nivel secundario de la Institución Educativa Francisco Antonio de Zela de Tacna, 2013. (Tesis de maestría). Universidad Nacional Jorge Basadre Grohmann- Tacna. Escuela de Posgrado. 
Mayer, J., \& Salovey, P. (1997). "What is emotional intelligence?", en Emotional Development and Emotional Intelligence,. New York Estados Unidos: Basik Books.

Menacho - López, J. C. (2010). Metodología de aprendizaje cooperativo como propuesta de innovación en la enseñanza de semiología general e interpretación de exámenes auxiliares. Tesis de maestría, Universidad Nacional Mayor de San Marcos, Lima, Perú.

Ministerio de Educación. (2017). Diseño Curricular Nacional.

Organización de las Naciones Unidas para la Educación, la Ciencia y la Cultura. (1998). Los docentes, la enseñanza y las nuevas tecnologías en Informe mundial sobre la educación. Madrid: Santillana/UNESCO.

Organización para la cooperación y desarrollo económico. (2011). Informe habilidades y competencias del siglo XXI para los aprendices del nuevo milenio en los países de la OCDE.

Piaget, J. (1950). The psychology of intelligence. New York- EE.UU: Harcourt.

Pujolás. (2009). La calidad en los equipos de aprendizaje cooperativo. Algunas consideraciones para el cálculo del grado de cooperatividad. En Revista de Educación, 349, mayo-agosto 2009, pp 225-239.

Roberts, R., Zeidner, M., \& Matthews, G. (2001). Does emotional intelligence meet traditional standards for an intelligence? Some new data and conclusions. Emotion, 196-231.

Salovey, P., \& Mayer, J. (1990). Emotional Intelligence. Imagination, Cognition and Personality, 9(3), 185-221.

Santos-Rego, M., Lorenzo-Moledo, M., \& Priegue-Caam. (2009). Aprendizaje cooperativo: práctica pedagógica para el desarrollo escolar y cultural Magis. Revista Internacional de Investigación en Educación, vol. 1, núm. 2, enero-junio, 2009, pp. 289- 30.

Traver, J. A. (2000). Trabajo cooperativo y aprendizaje solidario. Aplicación de la técnica puzzle de Aronson para la enseñanza y el aprendizaje de la actitud de solidaridad. Tesis doctoral, Universidad Jaume I, Castellón, España.

Trujillo, M., \& Rivas, L. (enero-junio de 2005). Orígenes, evolución y modelos de inteligencia emocional INNOVAR. INNOVAR. Revista de Ciencias Administrativas y Sociales, 15(25), 9.

Ugarriza, N. (2003). La evaluación de la inteligencia emocional a través del Inventario de BarOn (I-CE) en una muestra de Lima Metropolitana (2da. edición ed.). LimaPerú: Ediciones Libro Amigo.

Vivas, M., Chacón, E., \& Chacón, M. A. (enero-junio de 2010). Competencias socioemocionales autopercibidas por los futuros docentes. (U. d. Andes, Ed.) Educere, 14(48), 137-146.

Vygotsky, L. (1978). El desarrollo de los procesos psicológicos superiores. Barcelona: Crítica.

Zaccagnini, J. L. (2004). Qué es la inteligencia emocional. La elación entre pensamientos y sentimientos en la vida cotidiana. Madrid: Biblioteca Nueva. 